\title{
Optimierung der klinikinternen Ablauforganisation nach Einführung klinischer Pfade am Beispiel der Psoriasis
}

\author{
Implementing Clinical Pathways for Optimized Treatment of In-Patients with Psoriasis
}

Autoren

Institut
D. S. Müller, W. Ludwig-Peitsch, S. Goerdt, M. Goebeler

Klinik für Dermatologie, Venerologie und Allergologie, Universitätsmedizin Mannheim

\section{Bibliografie}

Dol http://dx.doi.org/

10.1055/s-0029-1215421

Online-Publikation: 27. 1. 2010

Akt Dermatol 2010; 36 :

115-120 ๑ Georg Thieme

Verlag KG Stuttgart · New York

ISSN 0340-2541

\section{Korrespondenzadresse}

Dr. med. Daniel S. Müller, MBA

Universitätsmedizin Mannheim

Medizinische Fakultät

Mannheim der Ruprecht-Karls-

Universität Heidelberg

Universitätsklinik für

Dermatologie, Venerologie

und Allergologie

Theodor-Kutzer-Ufer 1 -3

68167 Mannheim

daniel.mueller@haut.ma.uni-

heidelberg.de

\section{Zusammenfassung \\ $\nabla$}

Hintergrund: Gesundheitsökonomisch wünschenswert ist eine Verkürzung der Behandlungszeiträume bei gleichem oder sogar geringerem Personal- und Sachmitteleinsatz mit gleichzeitig besseren Behandlungsergebnissen. Für das Beispiel der stationären Psoriasis-Therapie mit Cignolin und UVB $311 \mathrm{~nm}$ bedeutet dies, die stationäre Verweildauer der Patienten bei mindestens gleichem Therapieerfolg zu verkürzen. Dieses Ziel kann durch Optimierung der bestehenden Therapieschemata realisiert werden.

Patienten und Methoden: Ende 2005 wurde in der Klinik für Dermatologie, Venerologie und Allergologie des Universitätsklinikums Mannheim mit der Entwicklung eines fachspezifischen Behandlungspfads „Psoriasis“ begonnen. Ziel war es, die Behandlungsqualität zu verbessern bzw. bei gleicher Ergebnisqualität die Verweildauern, orientierend an den DRG-Vorgaben zur mittleren Verweildauer, zu verkürzen. Die Effekte der Intervention wurden an insgesamt 408 stationären Patienten mit der Hauptdiagnose Psoriasis untersucht.

Ergebnisse: Nach Implementierung des Medizinischen Behandlungspfades „Psoriasis“ konnte die mittlere Verweildauer der Patienten von 18,61 Tagen im Jahr 2005 (Bezugsjahr vor Intervention) über 17,23 Tage für 2006 auf 16,69 Tage im Jahr 2007 gesenkt werden.

Schlussfolgerung: Medizinische Pfade sind ein geeignetes Mittel, um die Empfehlungen einer evidenzbasierten Leitlinie im klinischen Alltag umzusetzen. Die Behandlungsoptimierung bei gleichbleibend hoher oder sogar höherer Behandlungsqualität ist sinnvoll und kann Kosten und Verweildauer senken, jedoch nicht unbegrenzt.

\section{Einleitung}

\section{$\nabla$}

Das deutsche Gesundheitssystem, geprägt durch Solidarität und Subsidiarität, muss sich fortlaufend ändern und weiterentwickeln, um eine umfassende soziale Absicherung hinsichtlich Leistungsumfang, Versorgungsstruktur und Versorgungsqualität weiter gewährleisten zu können. Dieses System mit einem hohen Anspruchsniveau ist unterschiedlichen Zwängen ausgesetzt: Einerseits sind steigende Kosten durch demografische Faktoren, neue Technologien und steigende Erwartungen der Öffentlichkeit zu verzeichnen; andererseits sinken Beitragseinnahmen insbesondere bedingt durch die noch immer vergleichsweise hohe Arbeitslosigkeit sowie die sinkende Lebensarbeitszeit. Die weitere Finanzierbarkeit hängt nicht unerheblich von der Realisierung größerer Effizienz und stärkeren Wettbewerbs ab. Der zunehmende Wettbewerb zwischen den Leistungserbringern und der aus unterschiedli- chen Kostensteigerungen folgende Kostendruck zwingen die Krankenhäuser und Kliniken zur Überprüfung und Optimierung aller Strukturen und Prozesse. Die medizinische Leistungserbringung als eigentliche Kernkompetenz steht hierbei im Fokus der Sparmaßnahmen. Betrachtet man die Abläufe im Klinikalltag, so ist häufig eine gewisse Redundanz festzustellen, weiterhin ist einheitliches Qualitätsmanagement oft noch nicht etabliert. Der Zwang zur Kosteneffizienz erfordert den Einzug wirtschaftlicher Maßnahmen bei hoher und stetig steigender Behandlungsqualität in die tägliche Routine des ärztlichen Handelns. In vielen Fällen kann die Prozessoptimierung zum Wohle des Patienten hier mit einem höheren Grad an Standardisierung erreicht werden, was viele Kliniken zur Nutzung klinischer Behandlungspfade veranlasst. Diese können zu einer Verkürzung stationärer Behandlungszeiträume bei gleichem oder sogar geringerem Personal- und Sachmitteleinsatz mit gleichzeitig besseren Be- 
handlungsergebnissen beitragen [5,6]. Durch Kombination der Untersuchung der Parameter Zeit, Kosten und Qualität kann eine Prozessoptimierung nach betriebswirtschaftlicher Definition in die zwei verschiedenen Wirkungsrichtungen Steigerung der Prozesseffektivität („Die richtigen Dinge machen.“) und Verbesserung der Prozesseffizienz („Dinge richtig machen.“) erzielt werden. Übergeordnet kann dies als Erhöhung der Effektivität und Effizienz des klinischen Handelns beschrieben werden [10]. Ein Nutzen entsteht nur, wenn durch die Beschleunigung eines Teilprozesses die Verweildauer verringert werden kann oder freiwerdende Kapazitäten anderweitig genutzt werden können.

Die Einführung stationärer Behandlungspfade eignet sich insbesondere für Krankheitsbilder, die in einer ausreichend hohen Frequenz betreut werden und bei denen der gesamte Behandlungsverlauf weitestgehend plan- und vorhersehbar ist. Im dermatologischen Fachgebiet bietet sich hier beispielsweise die Psoriasis an, von der in Deutschland ca. 1,6 Millionen Patienten betroffen sind, davon ungefähr $20 \%$ von einer mittelschweren bis schweren Psoriasis, die stationär behandlungsbedürftig ist. Ausgehend von der Beobachtung, dass gerade für die Schuppenflechte die durch das DRG-System vorgegebenen Verweildauern deutlich von den tatsächlichen abweichen, entschieden wir uns im Jahr 2005, einen Behandlungspfad für dieses Krankheitsbild zu erarbeiten und ab 2006 zu implementieren. Neben dem Ziel einer Verringerung der stationären Aufenthaltsdauer und der damit verbundenen Kosten sollte auch sichergestellt werden, dass eine Konstanz der Therapieergebnisse unabhängig von den im Einzelfall für die Behandlung Verantwortlichen auf einem hohen Niveau gewährleistet ist. Schließlich sollten klinikinterne Abläufe insbesondere an den Schnittstellen zwischen ambulantem und stationärem Sektor sowie zwischen ärztlichem Dienst und Pflegedienst verbessert und um möglicherweise nicht zwingend indizierte medizinische Leistungen bereinigt werden.

Nachfolgend beschreiben wir die Einführung eines Behandlungspfads für die Psoriasis am Universitätsklinikum Mannheim und untersuchen die Auswirkungen auf die Behandlungsabläufe.

\section{Patienten und Methoden}

Die Analysen erfolgten an Patienten mit der Hauptdiagnose Psoriasis (ICD L40.0; zugeordnet den DRGs J61A und J61B), die sich in den Jahren 2005 bis 2007 an der Klinik für Dermatologie der Universitätsmedizin Mannheim einer stationären Therapie unterzogen. Alle 135 stationären Patienten mit der Hauptdiagnose Psoriasis des Jahres 2005 wurden einer Ist-Analyse (vor Einführung des Behandlungspfads) unterzogen, die 131 bzw. 142 Patienten der Folgejahre 2006 und 2007 in Bezug auf die erzielten Ergebnisse nach Umsetzung des Behandlungspfads untersucht. Der Großteil der Patienten fand sich in der DRG J61B wieder, nur eine Minorität wurde der DRG J61A zugeordnet. Unter die DRG J61A fielen im Jahr 200511 Fälle (8,1\%), im Jahr 200613 Fälle (9,9\%) und im Jahr 2007 nochmals 11 Fälle (7,7\%), durchschnittlich wurden dieser Diagnose 8,6\% der stationär behandelten Patienten zugeordnet, der DRG J61B somit 91,4\%. Für die Auswertungen wurde die Software KCC-MFI (Management-, Führungs- und Informationssystem, Dr. Kreysch Clinical Consulting $\mathrm{GmbH}$ ) herangezogen. Diese erlaubt die Analyse erbrachter Leistungen in Abhängigkeit vom Behandlungstag. Auf diese Weise kann für jeden individuellen Fall durch Auswertung der in den Leistungsdaten enthaltenen Zeitinformationen direkt und ohne zusätzlichen Erfassungsaufwand die Nachvollziehbarkeit des Medizinischen Pfa- des generiert werden. Die Zahlen werden in eine Graphik überführt, die Muster der Behandlungsprozesse (z.B. Abnahmezeitpunkt bestimmter Laborparameter, Röntgenuntersuchungen, etc.) durch separate Markierungen innerhalb einer Graphik verdeutlicht. Bei Bedarf kann die Summenkurve jeder DRG auf Einzelfälle heruntergebrochen werden, da die Datenbank eine Filterung (a) für alle erfassten Fälle, (b) nach Jahr und Monat, (c) nach Einzelfällen und (d) nach individuellen Parametern erlaubt. Mit diesem Instrumentarium ist es möglich, die einzelnen Leistungsmengen zu planen bzw. nachzuvollziehen. Aus der Summe der jeweiligen Leistungen (z.B. Labor, Liegetage, Radiologie, etc.) in den einzelnen DRGs ergeben sich die Gesamtleistungsmengen der jeweiligen Leistungserbringer.

\section{Ergebnisse}

$\nabla$

Nach Festlegung der Psoriasis als Zielindikation für einen zu implementierenden Behandlungspfad untersuchten wir in enger Zusammenarbeit mit dem Medizin-Controlling der Universitätsmedizin Mannheim zunächst die Ist-Situation des Jahres 2005. Es zeigte sich, dass die DRG-Vorgabe der mittleren Verweildauer eines stationär zu behandelnden Psoriasis-Patienten (ICD L40.0) für das Gesamtjahr bei 16,00 Tagen (Fallpauschalenkatalog zur Diagnose J61B) lag, die reale mittlere Verweildauer jedoch bei 18,61 Tagen. Somit lag hier eine Diskrepanz von 2,61 Tagen vor ( $)$ Abb. 1 a).

Diesen Befund nahmen wir zum Anlass, die bisherigen Abläufe in der Behandlung von Psoriasis-Patienten zu hinterfragen. Nach der Gliederung der Phasen der Optimierung des Behandlungsprozesses, implementierten wir unseren Medizinischen Behandlungspfad „Psoriasis“. Der Ablauf der Implementierung ist in - Tab. 1 zusammengefasst, die umgesetzten Maßnahmen sind in Tab. 2 dargestellt.

Das Pfad-Controlling und die regelmäßige qualitative Bewertung des Pfades durch die an der Behandlung Beteiligten sowie die Datenanalyse durch das Medizin-Controlling finden einerseits während der täglichen Visiten bzw. Patientenbetreuung statt, andererseits auch durch monatliche Analysen des Medizin-Controllings. Zusätzlich erfolgt ein laufendes Monitoring des Medizinischen Behandlungspfades mit Anpassungen an die jeweiligen Gegebenheiten und Weiterentwicklungen. Anhand der monatlich vorliegenden Zahlen und der Jahresauswertung für das Jahr 2006 überprüfen wir für das Jahr 2007 ein weiteres Optimierungspotenzial

- in Bezug auf den zeitlichen Ablauf,

- in Bezug auf die Leistungsmengen (Indikationen),

- in Bezug auf Standardisierungen und

- in Bezug auf sonstige Verbesserungen (wie z. B. die Einführung eines klinikzentralen Patientenmanagements inklusive stationärer Terminkoordination).

Unter anderem kann die Entlassung des Patienten am Abend des Tages erfolgen, der noch komplett als Behandlungstag genutzt wird. Hierdurch wird eine spätere Entlassung am Morgen des Folgetages mit einer weiteren zusätzlichen Übernachtung vermieden. Belegungsengpässe können somit leichter kontrolliert und mögliche freie Betten früher zur effektiveren Ressourcennutzung ausgeschöpft werden. Ein weiterer Punkt ist ein klares Komplikationsmanagement, wenn aufgrund von unerwünschten Nebenwirkungen kurzzeitig vom vorgegebenen Behandlungspfad abgewichen werden muss, was im jeweiligen Fall immer konsequent vom jeweils beteiligten Personal in der Patientenakte 
Abb. 1 a-c Verweildaueranalyse für hospitalisierte Patienten mit Psoriasis vulgaris (J61B) in den Jahren 2005 (a), 2006 (b) und 2007 (c).

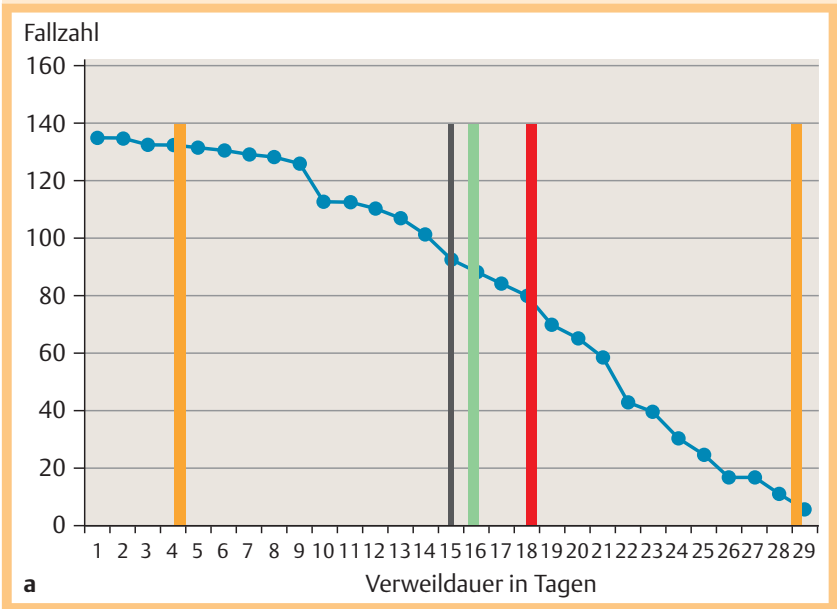

a Ausgangssituation 2005 (Abszisse: Verweildauer in Tagen, Ordinate: Fallzahl).

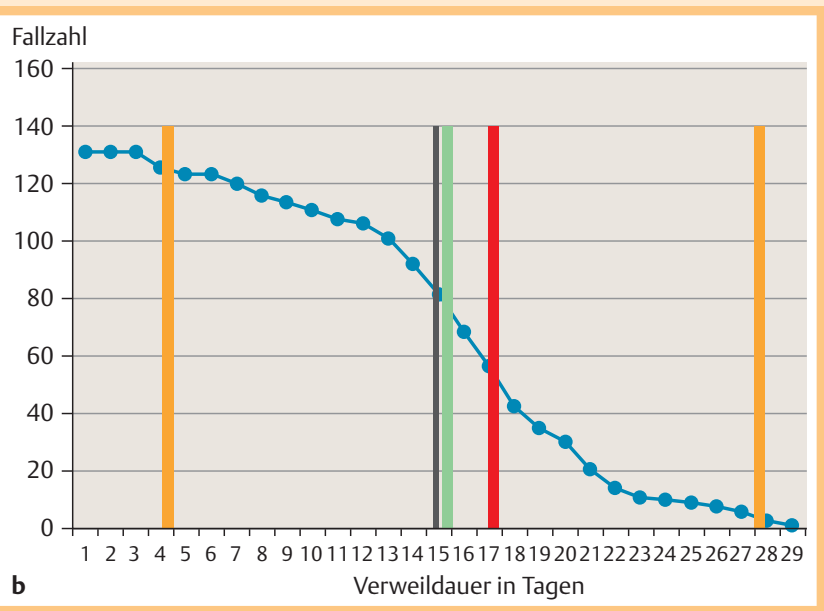

b Gesamtjahr 2006 (Abszisse: Verweildauer in Tagen, Ordinate: Fallzahl).

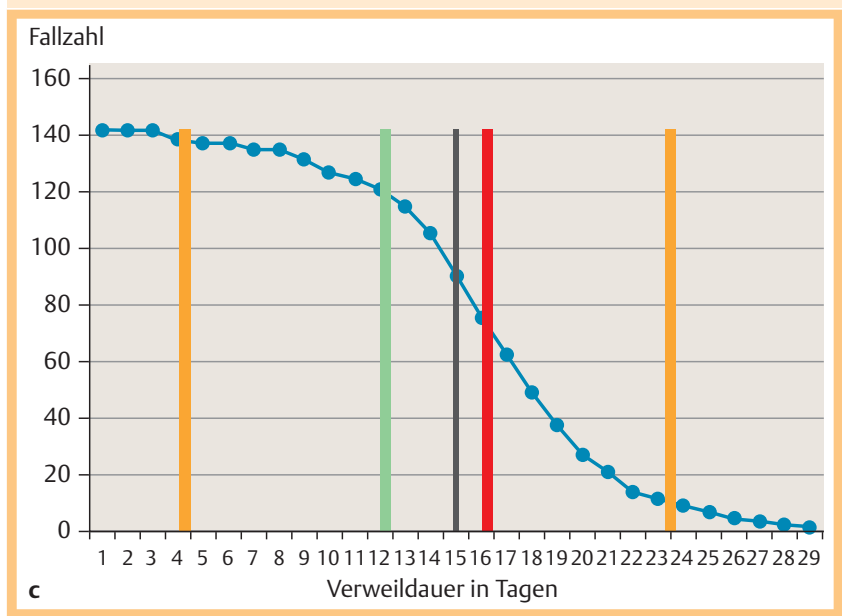

c Gesamtjahr 2007 (Abszisse: Verweildauer in Tagen, Ordinate: Fallzahl). Die gelben Balken im Diagramm verdeutlichen die untere bzw. die obere Grenzverweildauer aus dem jeweiligen Fallpauschalenkatalog der Diagnose J61B (schwere Erkrankungen der Haut, mehr als ein Belegungstag, Alter $>17$ Jahre oder mit komplexer Diagnose, ohne äußerst schwere CC [Komplikationen oder Komorbiditäten]). Die reale mittlere Verweildauer ist durch den roten Balken, die jeweilige DRG-Vorgabe der mittleren Verweildauer zur Diagnose J61B durch den grünen Balken symbolisiert. Der schmale schwarze Balken zeigt den Median. Der Kurvenverlauf stellt die Anzahl der Patienten dar, die nach x Tagen noch stationär sind. dokumentiert werden muss. Hier muss die Art der Abweichung mit entsprechender Begründung und Nennung des für die Abweichung Verantwortlichen festgehalten werden. Ziel ist eine schnelle Rückkehr auf den Medizinischen Behandlungspfad bei gleichbleibend hoher Behandlungsqualität unter Berücksichtigung der aufgetretenen Komplikationen.

Nach weitgehender Standardisierung dieses Gesamtprozesses und konsequenter Umsetzung im Jahr 2006 zeigten sich bei der Auswertung der Daten für das Jahr 2006 folgende Ergebnisse. Die Implementierung der o.g. Maßnahmen erlaubte eine Reduktion der realen mittleren Verweildauer stationär behandelter Psoriasis-Patienten auf 17,23 Tage. Allerdings wurde die DRGVorgabe für das Jahr 2006 ebenfalls gekürzt und betrug nur noch 15,50 Tage (Fallpauschalenkatalog zur Diagnose J61B). Die Diskrepanz zur verschärften Vorgabe konnte somit immerhin noch auf 1,73 Tage reduziert werden $(\boldsymbol{\otimes} \mathbf{A b b} \mathbf{1} \mathbf{1} \mathbf{b})$.

Im Jahr 2007 gelang es, unter konsequentem Pfadmanagement die reale mittlere Verweildauer stationär behandelter PsoriasisPatienten auf nunmehr 16,69 Tage zu senken. Hier wurde vor allem nochmals die konsequente Einhaltung der oben genannten Maßnahmen in ihren Zeitfenstern von der Arbeitsgruppe geprüft. Schnittstellenproblematiken (vor allem der Dokumentenaustausch) zwischen Station und Ambulanz wurden durch Einführung eines klinikinternen Patientenmanagements optimiert und die Terminvereinbarung mit anderen Disziplinen (Radiologie, Nuklearmedizin, etc.) in den ambulanten Bereich vorverlagert. Die DRG-Vorgabe wurde für das Jahr 2007 jedoch auch nochmals deutlich auf 12,60 Tage (Fallpauschalenkatalog zur Diagnose J61B) gekürzt. Aufgrund dieser maximalen Kürzung der Vorgabe erhöhte sich die Diskrepanz zur tatsächlichen Verweildauer im Jahr 2007 auf 4,09 Tage ( $\bullet$ Abb. 1 c und Abb. 2).

Diese Kürzung wurde jedoch für das aktuelle Jahr 2008 zumindest auf 13,00 Tage nach oben korrigiert. Der Median lag für alle untersuchten Jahre 2005, 2006 und 2007 bei 15 Tagen.

\section{Diskussion \\ $\nabla$}

Bei Einführung, Nutzung und Optimierung von Medizinischen Behandlungspfaden (Medical Pathways) bleibt festzuhalten, dass ein Nutzen im Sinne einer Effizienzsteigerung nur entsteht, wenn durch die Beschleunigung eines Teilprozesses die Verweildauer verringert werden kann oder freiwerdende Kapazitäten anderweitig genutzt werden können.

Bei der Prozessoptimierung kann daher ein Netzplan mit Zerlegung des gesamten Klinikaufenthaltes in einzelne Teilprozesse von Nutzen sein. Ein solcher ist definiert als Verfahren zur Analyse, Beschreibung, Planung, Steuerung und Überwachung von Abläufen, wobei Zeit, Kosten, Einsatzmittel bzw. Ressourcen berücksichtigt werden können. Netzpläne bieten einen Gesamtüberblick über den Hauptprozess oder den ganzen Behandlungspfad und stellen somit sicher, dass das strategische Ziel, hier speziell die Reduzierung des notwendigen Klinikaufenthaltes bei gleichbleibend hoher oder sogar höherer Behandlungsqualität und entlassfähigem Hautbefund, nicht aus den Augen verloren wird. Anhand der einzelnen Teilprozesse sollte dann regelmäßig überprüft werden, wo noch Verbesserungspotenzial vorhanden oder durch bereits durchgeführte Optimierungen neu entstanden ist. Die Kosten einer Verkürzung einzelner Arbeitsschritte werden dem Nutzen einer früheren Beendigung eines Prozesses gegenübergestellt. Konkret entstehen durch z.B. eine Schnittstellenoptimierung keine weiteren Kosten, die dadurch erreichte Verkür- 
Tab. 1 Phasen der Optimierung des Behandlungsprozesses der Psoriasis an der Klinik für Dermatologie der Universitätsmedizin Mannheim.

Initialisierung
- Festlegung der Indikation (Psoriasis)
- Bestimmung der Pfadverantwortlichen und Einsetzung einer
Arbeitsgruppe (mit Ärztlichem und Pflegedienst)
Analyse
- Identifikation der Schnittstellen/der benötigten Kolleginnen und
Kollegen
- Analyse der Ist-Situation (Schwachstellenanalyse; gemeinsam mit
dem Medizin-Controlling)
Entwicklung des Pfades
- Entwurf des Soll-Prozesses und Abgleich mit der Ist-Situation
- Klärung der Veränderungsnotwendigkeiten
Einführungsentscheidung
- Verabschiedung durch die Arbeitsgruppe
- Verabschiedung durch die Abteilungsleitung
Pfad-Controlling
- Regelmäßige qualitative Bewertung des Pfades durch die an der
Behandlung Beteiligten
- Datenanalyse durch das Medizin-Controlling
Anpassung
- Bei Bedarf Anpassung und Weiterentwicklung

zung der Liegezeit eröffnet aber Potenzial zur Nutzung früher freiwerdender Ressourcen, was monetär durch eine Belegungsoptimierung geltend gemacht werden kann.

Die Schwierigkeit liegt darin, dass nicht alle zukünftigen Vorteile als Kostenersparnisse oder Erträge messbar sind. So können z.B. die finanziellen Auswirkungen einer erhöhten Patienten- oder Mitarbeiterzufriedenheit allenfalls geschätzt werden.

Neben den Kosten wird geprüft, ob die Qualität der Behandlung stabil geblieben ist oder sogar verbessert werden konnte. Es erfolgt also eine vergleichende Messung der Prozessleistungstrans- parenz nach den zu Projektbeginn definierten Kennzahlen. Dies sollte bei dem Krankheitsbild „Psoriasis“ anhand der bewährten Patienten-Evaluationsinstrumente wie PASI (Psoriasis Area and Severity Index) und DLQI (Dermatology Life Quality Index) erfolgen, was einen Ausgangspunkt zur Überprüfung in weiteren Studien bietet. Sicherlich muss speziell das Instrument DLQI bei Krankenhausaufenthalten per se kritisch hinterfragt werden, da bestimmte Parameter hier während eines Krankenhausaufenthaltes nicht messbar sind und somit die Gefahr der Verfälschung des Ergebnisses besteht. In unserem Fall gab es für das Jahr 2005 keine historischen Daten zu Erfolgs- bzw. Qualitätsparametern (PASI, DLQI). Zusätzlich lagen Verlaufskontrollen nach der Entlassung nicht vollständig vor, da sich nur ein Teil der Patienten ambulant in der Klinik wiedervorstellt. Diese Schnittstellenproblematik zwischen den Kliniken und den niedergelassenen Dermatologen bzw. anderen nachbehandelnden Fachgruppen bietet weiteres Optimierungspotenzial. Ein klares Nachsorgeschema mit Überprüfung der Erfolgs- bzw. Qualitätsparameter sollte zukünftig von allen Beteiligten angestrebt und kontrolliert werden. Der Nutzen eines Behandlungspfades liegt zum einen in einer koordinierten Vorgehensweise und damit Bündelung der Kräfte auf eine gemeinsame Zielsetzung, zum anderen in der Optimierung der bereits erwähnten vielfältigen Schnittstellen - hier speziell der ambulanten und der stationären Versorgung - im Konsens der verantwortlichen Bereiche. Eine koordinierende berufs- und hierarchieübergreifende Managementstruktur in Form des Medizin-Controllings begleitet und analysiert die Umsetzung des Behandlungspfades in monatlichen Abständen. Die Verantwortung für den Pfad - und dies entkräftet auch teilweise wieder das Argument der Einschränkung der Behandlungsfreiheit - inklusive der Dokumentation bei medizinisch notwendiger Abweichung vom Soll-Pfad und das aktuelle Pfad-Controlling muss aber bei den behandelnden Ärztinnen und Ärzten liegen, da diese
- Bestimmung der kompletten Laborroutine am Tag der stationären Aufnahme; weitere Laborkontrollen nur nach medizinischer Notwendigkeit, um vormals übliche routinemäßige Doppeluntersuchungen zu vermeiden.

- Frühestmöglicher Behandlungsbeginn durch zeitliche Optimierung der Anmeldeformalitäten am Aufnahmetag; der Modus wird den Patienten bereits bei der Planung der stationären Aufnahme in der Ambulanz durch das Patientenmanagement erläutert.

- Alle nicht persönlich zu erledigenden Aufnahmeformalitäten werden vorab bereits am Tag der Entscheidung zur stationären Aufnahme von dem zentralen Patientenmanagement (eingeführt ab 2007) in der Hautklinik vorgenommen, um Verzögerungen am Aufnahmetag zu vermeiden.

- Standardisierte Befunddokumentation (inkl. PASI und DLQI) und Festlegung der stationären Therapie durch Assistenz- bzw. Oberarzt bereits in der Ambulanz am Tag der Vorstellung. Abschuppende Maßnahmen werden, soweit möglich, bereits vor stationärer Aufnahme eingeleitet, die stationäre Therapie (üblicherweise Cignolin [Dithranol-]Lokaltherapie und UVB 311 nm-Lichttherapie) inkl. ihrer Anwendungsmodalitäten (z. B. Dosierungen) auf standardisierten Laufzetteln dokumentiert und an die Stationen übermittelt. Somit ist ein sofortiger Therapiebeginn am Aufnahmetag bereits vormittags nach Erledigung der Aufnahmeformalitäten ohne weitere Zeitverzögerung möglich.

- Standardisierung der Abläufe: Erfolgte bzw. vorgegebene Therapien werden auf dem Plan zum Medizinischen Behandlungspfad mit Handzeichen abgezeichnet, nur in Ausnahmefällen, die ausführlicher dokumentationspflichtig sind, wird hiervon abgewichen.

- Ggf. erforderliche Untersuchungen wie z. B. Röntgenuntersuchungen oder Knochenszintigrafie zur Abklärung einer Psoriasisarthritis werden bereits bei Regelung der Aufnahmeformalitäten durch das Patientenmanagement im Vorfeld terminiert, um Verzögerungen während des stationären Aufenthaltes weitestgehend zu vermeiden.

- Einweisung des Patienten durch ärztliches und Pflegepersonal bezüglich ambulant fortzuführender Therapien nach Entlassung aus stationärer Behandlung (z. B. bezüglich der Handhabung harnstoffhaltiger Externa sowie anderer Lokaltherapeutika, die nach der Entlassung durch den Patienten selbst appliziert werden sollen).

- Die Entlassung der Patienten wird regelhaft am Abend eines vollen Behandlungstages angestrebt; somit ist der Entlasstag noch voller Behandlungstag, wodurch eine weitere Kliniknacht vermieden werden kann.

- Ein konkreter Folgeplan zur Anschlussbehandlung nach Entlassung mit weiteren Terminen beispielsweise zur Lichttherapie bzw. zur Behandlungserfolgskontrolle in der Sondersprechstunde „Psoriasis“ wird den Patienten am Entlassungstag ausgehändigt, um eine optimale Nachbehandlung im ambulanten oder tagesklinischen Bereich zu gewährleisten.
Tab. 2 Parameter, die im Rahmen der Optimierung des Behandlungsprozesses der Psoriasis im Behandlungspfad berücksichtigt und umgesetzt wurden. 


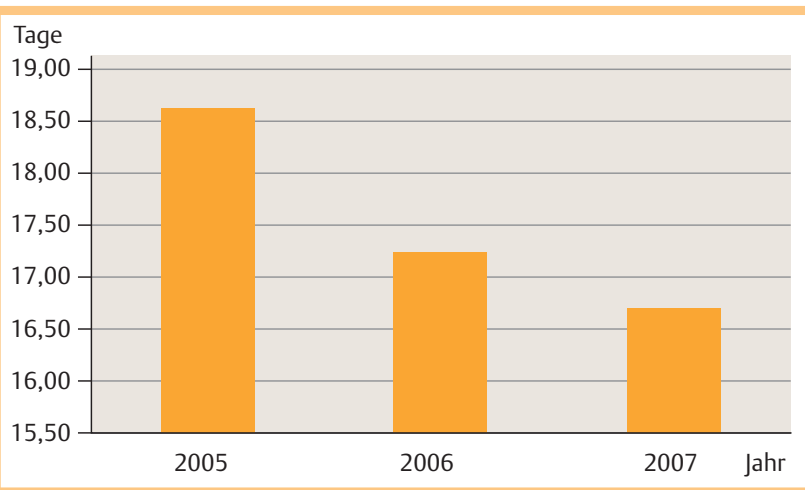

Abb. 2 Mittlere Verweildauer hospitalisierter Psoriasis-Patienten in den Jahren $\mathbf{2 0 0 5}$ bis 2007. Berücksichtigt wurden Psoriasis-Fälle mit den Diagnosen aus dem DRG-Fallpauschalenkatalog J61A und J61B (Abszisse: Jahre, Ordinate: Tage).

den Gesamtbehandlungsprozess generieren und gestalten. Der Vorteil in der Standardisierung liegt vor allem darin, dass bei hoher Personalfluktuation das Risiko gesenkt wird, dass durch sehr individuelle Behandlungen verschiedener Leistungsträger eines Behandlungsteams unterschiedliche qualitative Ergebnisse resultieren. Daher verlangen auch die Qualitätsrichtlinien der Joint Commission on Accreditation of Healthcare Organizations (JCAHO) eine solche Standardisierung (http://www.jcrinc.com/fpdf/pubs/ pdfs/JCReqs/JCP-09-07-S3.pdf).

Der Medizinische Pfad ist zusätzlich ein geeignetes Mittel, um die Empfehlungen einer evidenzbasierten Leitlinie im klinischen Alltag umzusetzen. Die Konzepte ergänzen sich gegenseitig, indem die evidenzbasierte Leitlinie eine umfassende nationale Rahmenempfehlung gibt, insbesondere auf der Ebene der Entscheidungsunterstützung, die anhand eines Medizinischen Pfades auf die Umstände der medizinischen Praxis adaptiert werden kann. So können über die evidenzbasierte Medizin hinaus auch lokale Ziele und Besonderheiten berücksichtigt werden.

Müller et al. berichten in ihren „Fakten und Analysen“ zur G-DRG Version 2008 [9], dass aufgrund der starken Abhängigkeit der kalkulierten Kosten von der Verweildauer unter anderem auch die sinkenden Verweildauerzahlen in dermatologischen Kliniken mitverantwortlich für einen Rückgang der DRG-Bewertungen sind. Dies bestätigt sich in der kontinuierlichen Kürzung der DRG-Vorgabe für die mittlere Verweildauer, in unserem Beispiel - wie dargelegt - bis auf den Wert von 12,60 Tagen (Fallpauschalenkatalog zur Diagnose J61B) für das Jahr 2007. Hier schließen wir uns der Bewertung von Müller et al. [9] an, dass „der Rückgang der Verweildauern bzw. die DRG-Vorgaben hierzu kein Prozess sein darf, der stetig fortschreitet, sondern sich selbst limitieren muss, da die Grenzen notwendiger, zweckmäßiger und wirtschaftlicher medizinischer Versorgung nicht unterschritten werden dürfen“. Weiterhin müssen bei der Interpretation der Vorgabe der mittleren Verweildauer durch das Institut für das Entgeltsystem im Krankenhaus (InEK) sicher auch die unterschiedlichen Schweregrade der Psoriasis-Fälle an den verschiedenen Krankenhäusern diskutiert werden. Eine Diskrepanz tritt auch in der Nachhaltigkeit der jeweiligen Therapien auf. Selbstverständlich lässt sich ein schnelleres Ansprechen auf die Therapie und somit eine niedrigere Verweildauer bei der Psoriasis mit einer SteroidTherapie erreichen, jedoch ist hier auch wieder mit einem schnelleren Rezidiv/Rebound zu rechnen, was einen baldigen erneuten kostenintensiven stationären Aufenthalt zur Folge haben kann. Die sinnvolle Behandlungsoptimierung muss immer auch das medizinisch vertretbare Ergebnis neben der Kosten- und Verweildauersenkung im Auge haben. Eine unbegrenzte Regelung nach unten an dieser Stellschraube ist somit weder praktikabel noch erstrebenswert.

Im Ausblick zur Entwicklung weiterer Medizinischer Behandlungspfade für klar zu definierende Krankheitsbilder und Therapieschemata spielt die zunehmende Lebenserwartung der Bevölkerung mit höherem stationärem Patientenaufkommen in Kombination mit Multimorbidität eine wichtige Rolle. Ohne Optimierung der Abläufe nimmt die Schwierigkeit zu, eine qualitativ hochwertige Patientenversorgung innerhalb der vorgegebenen Grenzverweildauern zu gewährleisten.

\section{Fazit für die Praxis \\ $\nabla$}

Am Beispiel der stationären Therapie der Psoriasis vulgaris wird dargelegt, dass die Implementierung klinischer Behandlungspfade zu einer Verkürzung stationärer Verweildauern beitragen kann und damit die (gerade für die Psoriasis besonders ausgeprägte) Diskrepanz zu den DRG-Vorgaben verbessern hilft.

\section{Interessenkonflikt \\ $\nabla$}

Es besteht kein Interessenkonflikt. Der korrespondierende Autor versichert, dass keine Verbindungen mit einer Firma, deren Produkt in dem Artikel genannt ist, oder einer Firma, die ein Konkurrenzprodukt vertreibt, bestehen. Die Präsentation des Themas ist unabhängig und die Darstellung der Inhalte produktneutral.

\section{Abstract}

\section{Implementing Clinical Pathways for Optimized Treat- ment of In-Patients with Psoriasis \\ $\nabla$}

Background: From a health economic perspective, it is desirable to reduce time periods of hospitalization but to simultaneously maintain or even improve the quality of treatment. Studying combined cignolin/UVB $311 \mathrm{~nm}$ treatment of hospitalized patients with moderate to severe plaque-type psoriasis we analyzed periods of hospitalization before and after implementation of a specific clinical pathway.

Patients and methods: At the end of 2005, a clinical pathway for optimized treatment of in-patients with psoriasis was implemented at the Department of Dermatology, University Hospital Mannheim, in order to improve quality and to reduce hospitalization times thus meeting the requirements of the DRG provisions. A total of 408 patients with a main diagnosis of psoriasis were analyzed before and after implementation of the clinical pathway in the years 2005 to 2007.

Results: After implementing a medical treatment pathway for psoriasis the average period of hospitalization could be reduced from 18.61 days in 2005 to 17.23 days in 2006 and 16,69 days in 2007.

Conclusion: The medical pathway is an appropriate measure to transfer the recommendations from an evidence-based guidance into daily clinical routine. The optimization of treatment next to a constantly high or even higher treatment quality is very reasonable since it allows reduction of costs and hospitalisation periods; however, not to an unlimited extent. 


\section{Literatur}

1 Coffey RJ, Richards JS, Remmert CS et al. An introduction to critical paths. Quality Management in Health Care 1992; 1 (Vol. 1): $45-54$

2 Dy SM, Garg PP, Nyberg D et al. Are critical pathways effective for reducing postoperative length of stay? Med Care 2003; 41: 637-648

3 Ewers M, Schaeffer D. Case Management in Theorie und Praxis. Bern: Hans Huber, 2000

4 Giffin M, Giffin R. Critical pathways produce tangible results. Health Care Strategic Management 1994; 7 (Vol. 12): 17-23

5 Korycan T. Tips from the Front Line. Achieving Cost and Quality Benefits in Critical Pathway Implementation. Quality Management in Health Care 1995; 2 (Vol. 3): $71-73$

6 Küttner T, Wiese M, Roeder $N$. Klinische Behandlungspfade - Teil 1: Hohe Qualität zu niedrigen Kosten - ein unlösbarer Zielkonflikt? Pflegezeitschrift 2005; 3: 176-179

7 Küttner T, Wiese M, Roeder N. Klinische Behandlungspfade - Teil 2: Der Pfad ist das Ziel - und eine optimierte Entwicklungsmethodik. Pflegezeitschrift 2005; 4: $252-256$
8 Melbert RB, Kimmins MH, Isler JT et al. Use of a critical pathway for colon resections. J Gastrointest Surg 2002; 6: 745-752

9 Müller ML, Forschner A, Wenke A et al. G-DRG Version 2008: Fakten und Analysen. JDDG 2008; 6: 483-491

10 Roeder N, Hensen P, Hindle D et al. Instrumente zur Behandlungsoptimierung: Klinische Behandlungspfade. Der Chirurg 2003; 12: 1149 1155

11 Roeder N, Hindle D, Loskamp $N$ et al. Frischer Wind mit klinischen Behandlungspfaden (I): Instrumente zur Verbesserung der Organisation klinischer Prozesse. Das Krankenhaus 2003; 1: 20-27

12 Roeder N, Hindle D, Loskamp $N$ et al. Frischer Wind mit klinischen Behandlungspfaden (II): Instrumente zur Verbesserung der Organisation klinischer Prozesse. Das Krankenhaus 2003; 2: 124-130

13 Spath $P$. How to manage clinical path variance data. Hosp Case Manag 2000; 8: $77-79$

14 Takegami K, Kawaguchi Y, Nakayama H et al. Impact of a clinical pathway and standardization of treatment for acute appendicitis. Surg Today 2003; 33: $336-341$ 